\title{
Effect of ethanol infusion on the ultrastructure of human myocardium
}

\author{
H. KLEIN \\ M.D. \\ D. HARMJANZ \\ M.D. \\ Department Innere Medizin, Medizinische Hochschule, Hannover and \\ Abteilung Elektronenmikroskopie, Anatomisches Institut, Medizinische Hochschule, Hannover*
}

\begin{abstract}
Summary
To study the influence of a moderate dose of ethanol on the ultrastructure of myocardium in patients with heart disease, a $7 \cdot 5 \%$ solution of ethanol was infused in six patients with myocardial and valvular disease, who underwent diagnostic cardiac catheterization. Before and after infusion of ethanol an endomyocardial biopsy was performed and the specimens were examined by electron microscopy. After ethanol all six patients revealed alterations in the mitochondrial structure and three of them also showed significant dilatation of the sarcoplasmic reticulum. Cardiac output increased in three of the patients, whereas in two patients a decrease of cardiac output was measured.

The results suggest that the infusion of even small amounts of ethanol is able to provoke structural alterations of myocardial cell organelles and, therefore, possible impairment of myocardial function.
\end{abstract}

OWING to the growing consumption of alcohol our endomyocardial biopsies are also concerned with alcoholic cardiomyopathy. There are already a number of authors, such as Alexander (1966a, b, 1967a, b), Ferrans et al. (1965), Burch and DePasquale (1969), Burch and Giles (1971), Burch et al. (1 971) and others, who have investigated the effect of alcohol in myocardium of animals as well as in man.

It is not our intention to add another paper to these excellent studies, but we believe that our recent observations may be of interest. We have emphasized the question as to what kind of ultrastructural changes occur if a moderate dose of ethanol is infused to patients with known cardiac disease, especially when solutions containing ethanol are employed for caloric replacement.

\section{Selection of patients and method}

In six patients who underwent diagnostic cardiac catheterization because of myocardial and valvular disease a $7.5 \%$ solution of ethanol in $380 \mathrm{ml} \mathrm{NaCl}$ $0.9 \%$ was infused over $30 \mathrm{~min}$ through the femoral vein. Previous consent for this examination was obtained from all patients.

* Supported by Deutsche Forschungsgemeinschaft.
Endomyocardial biopsy with a Konno bioptome (Konno and Sakakibara, 1963) was carried out from the right ventricle before and immediately after ethanol infusion. At the time of the second biopsy the actual concentration of blood alcohol was determined from a brachial vein. Two to three biopsies were performed on each patient before and after ethanol administration.

The specimens were fixed in $2.5 \%$ glutaraldehyde and $2 \%$ formaldehyde in $0.1 \mathrm{~mol}$ cacodylate buffer at $\mathrm{pH} 7 \cdot 3$, post-fixed in $1 \%$ osmium tetroxide in Millonig's phosphate buffer and embedded in Epon 812. Semifine sections were stained with toluidine blue and in some specimens PAS-reaction was performed. Thin sections were stained with lead citrate and examined with an electron microscope 'Siemens la'.

There were two chronic alcoholics, three cases of valvular disease and a woman with suspected myocarditis. All investigated patients suffered from cardiac decompensation before admission but were compensated at the time of catheterization. They showed cardiomegaly and electrocardiographic abnormalities.

\section{Results}

The two chronic alcoholics showed high cardiac output before biopsy and a further increase of cardiac output was measured after ethanol infusion.

Light microscopic examination showed signs of hypertrophy with enlarged diameter of the muscle fibres, polymorphic nuclei, interstitial fibrosis, patchy myofibril destruction and vacuoles which contained glycogen as shown by PAS-positive reaction.

Electron microscopic examination of the two alcoholics revealed normal mitochondria before ethanol infusion, whereas swelling of mitochondria was evident after ethanol. The mitochondrial matrix was less dense and there was a significant loss of cristae. The sarcoplasmic reticulum showed abnormal dilatation after ethanol (Fig. 1).

An accumulation of lipid droplets, especially 

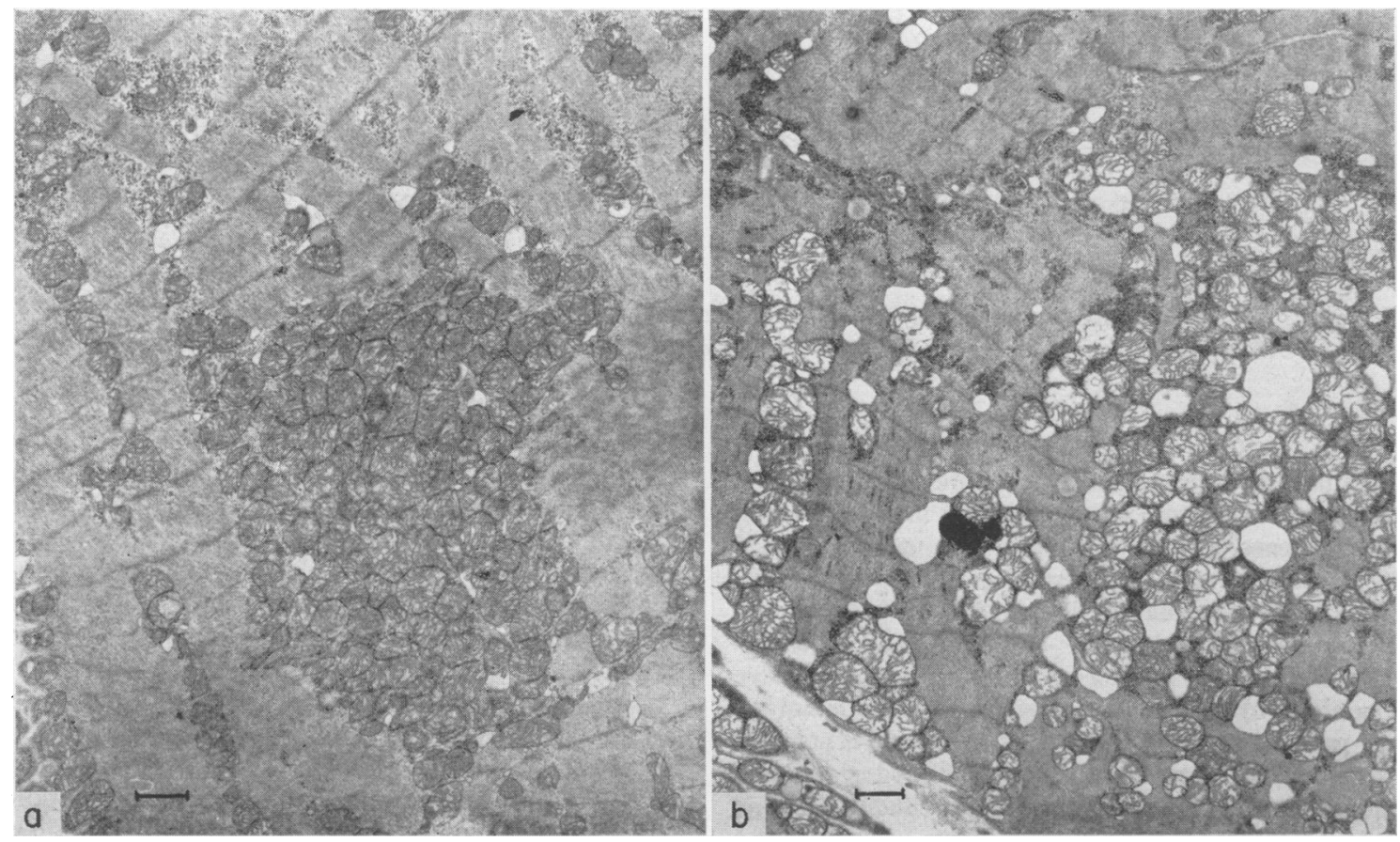

FIG. 1. Biopsy from a chronic alcoholic. (a) Before ethanol infusion, showing normal mitochondria; (b) after ethanol swelling of mitochondria is evident, mitochondrial matrix is less dense. Sarcoplasmic reticulum shows abnormal dilatation. Both specimens reveal abundant glycogen granules.

under the sarcolemma was observable in the chronic alcoholics. These lipid droplets were often surrounded by glycogen granules. Abundant glycogen granules were the most characteristic finding in the myocardium of alcoholics, but the content of glycogen seemed not to be altered after ethanol.

A patient with aortic regurgitation revealed an increase of cardiac output after ethanol. Normal mitochondria and sarcoplasmic reticulum were noted before ethanol administration, whereas a marked swelling of sarcoplasmic reticulum and a less dense mitochondrial matrix were found after ethanol infusion.

A woman with severe bivalvular disease had a low cardiac output just before ethanol administration and a dangerous fall of arterial blood pressure was measured during the period of ethanol infusion. Therefore, no reliable haemodynamic data were obtained. No abnormality in mitochondrial structure was seen before ethanol, yet swollen mitochondria with less dense matrix and loss of cristae were found after ethanol (Fig. 2).

In a patient with compound aortic valve disease and who had a decrease in cardiac output after ethanol, significant alteration in mitochondrial structure was also observable after ethanol. Most of the cristae seemed fragmented and the characteristic zig-zag course had disappeared.

In the case of suspected chronic myocarditis, a decrease of cardiac output was measured after ethanol. The most important alteration after the infusion was found in the severe dilatation of the sarcoplasmic reticulum. The tubules contained flocky and partly granular structures. Alteration of the mitochondria could be seen, but it was not so remarkable as in the other patients.

In hypertrophied myocardium it was interesting to observe the increase of subsarcolemmal sarcoplasm with augmented amount of ribosomes and glycogen granules but not yet visible myofilaments. This seems characteristic for longitudinal growth of the muscle cells as it is described by Meessen (1971) in stepwise dove-tailing of the intercalated discs (Fig. 2).

In all our investigated patients no alteration in the amount of intramitochondrial granules could be observed after ethanol. There was no general swelling of capillary endothelium, but in some specimens a 

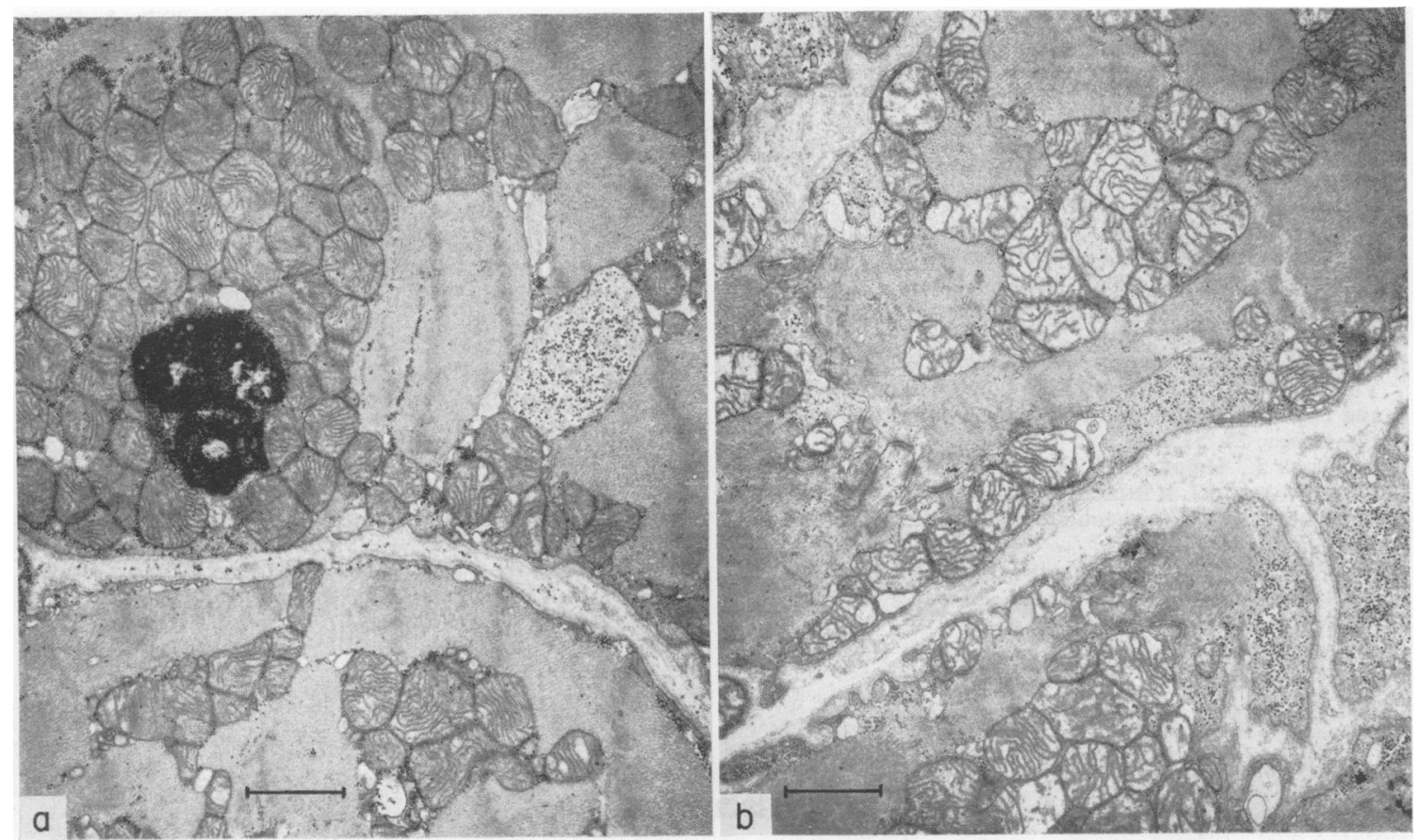

Fig. 2. Biopsy from a patient with bivalvular disease and hypertrophy. (a) Before ethanol infusion no abnormality in mitochondrial structure is seen; great lipofuscingranule in a cluster of mitochondria; (b) after ethanol: swelling of mitochondria, enlighted matrix and loss of cristae. Note the areas of subsarcolemmal sarcoplasm without myofilaments indicating longitudinal growth in hypertrophy.

focal oedema of endothelial cells was noted. The nuclei and intercalated discs showed no alteration which could be referred to ethanol infusion.

\section{Discussion}

We have seen alterations of myocardial cell organelles after the infusion of a moderate dose of ethanol. The mean alcohol concentration in blood at the time of the second biopsy was $0.38 \mathrm{~g} \%$, almost equal to a concentration obtained after the consumption of two or three beers or of a glass of whisky.

Although some areas of the investigated myocardium revealed less severe alterations, they were present in all six patients. From these observations it may be concluded that alteration in myocardial ultrastructure can be related to the influence of ethanol.

Our investigation was also intended to support the results of the haemodynamic findings of Gould et al. (1971) who described a decrease of cardiac index and stroke index and widening of arteriovenous oxygen difference in patients with cardiac disease after oral application of $2 \mathrm{oz}$ whisky.

Although the cause of mitochondrial swelling and structural alteration after alcohol administration has still to be investigated, one reason for this swelling can probably be found in the observations of Lenaz et al. (1971) who described how alcohol depleted mitochondrial membranes of phospholipids because of ruptured lipid protein bonds. He measured an inhibition of ATPase activity and oxidative phosphorylation. Similar observations were made by Gvozdjakova et al. (1971, 1972). Therefore it is possible that alterations to mitochondrial and sarcoplasmic reticulum membranes are able to produce mitochondrial swelling, disturbance in mitochondrial calcium binding and uptake mechanism (Pachinger et al., 1973) and loss of enzymes necessary to the oxidative metabolism.

Our observations do suggest that the infusion of solutions containing alcohol as caloric replacement is not advisable in patients with heart disease owing to the possible impairment of the myocardial function.

\section{References}

Alexander, C.S. (1966a) Idiopathic heart disease. 1. Analysis of 100 cases, with special reference to chronic alcoholism. American Journal of Medicine, 41, 213. 
Alexander, C.S. (1966b) Idiopathic heart disease. 2. Electron microscopic examination of myocardial biopsy specimens in alcoholic heart disease. American Journal of Medicine, 41, 229.

AleXANDER, C.S. (1967a) Electron microscopic observations in alcoholic heart disease. British Heart Journal, 29, 200.

AleXANDER, C.S. (1967b) Alcohol and the heart. Annals of Internal Medicine, 67, 670.

Burch, G.E. \& DePasquale, N.P. (1969) Alcoholic cardiomyopathy. American Journal of Cardiology, 23, 723.

BURCH, G.E. \& GILES, T.D. (1971) Alcoholic cardiomyopathy. Concept of the disease and its treatment. American Journal of Medicine, 50, 141.

Burch, G.E., Colcolough, H.L., Harb, J.M. \& Ching, YA Tsui (1971) The effect of ingestion of ethylalcohol, wine and beer on the myocardium of mice. American Journal of Cardiology, 27, 522.

Ferrans, V.J., Hibbs, R.G., Weilbaecher, D.G., Black, W.C., Walsh, J.J. \& BuRCh, G.E. (1965) Alcoholic cardiomyopathy. A histochemical study. American Heart Journal, 69, 748.

Gould, L., ZAHiR, M., De Martino, A. \& Gomprechr, R.F. (1971) Cardiac effects of a cocktail. Journal of the American Medical Association, 218, 1799.
Gvozdjakova, A., Bada, V., Niederland, T.R. \& GvozDJAK, J. (1971) Contribution à l'effet aigu de l'éthanol sur le métabolisme du myocarde. Cor et vasa, Praha, 13, 229.

Gvozdjakova, A., Bada, V., KRUty, F., Niederland, T.R. \& GvozdjAK, J. (1972) Dynamics of metabolic changes following administration of ethanol. Cor et vasa, Praha, 14, 239.

Konno, S. \& SAKakibara, S. (1963) Endomyocardial biopsy. Diseases of the Chest, 44, 345.

Lenaz, G., Parenti-Castelli, G., Monsigni, N. \& SilveSTRINI, M.G. (1971) Effects of alcohols on the functional organization of the inner mitochondrial membrane. Bioenergetics, 2, 119.

MeEssen, H. (1971) Structural bases of myocardial hypertrophy. British Heart Journal, 33, Supplement, 94.

Millonig, G. (1961) Advantage of a phosphate buffer for $\mathbf{0 5 0}_{4}$ solution in fixation. Journal of Applied Physics, 32, 1367.

Pachinger, O., Mao, J., Tillmanns, H. \& Bing, R.J. Mitochondrial function and excitation-contraction coupling in the development of alcoholic cardiomyopathy. VIth Annual Meeting of the International Study Group for Research in Cardiac Metabolism, Freiburg, Sept. 1973.

\section{Discussion}

Chairman: Any questions or comments to Dr Klein?

Professor MUIR: Could I just ask one question on method? This morning we talked a great deal about the reproducibility of samples from the myocardium and the number of samples that needed to be taken was stressed. Were these the results of one sample before alcohol and one after, or how many biopsies were done before the alcohol infusion, and how many after?

DR KLEIN: We always performed three or four biopsies before and four after alcohol infusion. All specimens were fixed in the same manner. We believe that four samples are sufficient to reveal possible alterations of the myocardium.

DR BRACHFELD: Could I ask you, Dr Klein, whether you have any evidence to support your last sentence that you believe these changes to be reversible, either in man or in animal?

DR KLEIN: Unfortunately that is hypothesis, because we are not able to take another biopsy $2 \mathrm{hr}$ or a day later.

DR BRACHFELD: You have not done this.

DR SCHWARTZ: I think this is quite interesting, but the last sentence suggested that we should not give alcohol even, I gather, an acute shot of alcohol to somebody suffering from heart disease. I assume this is based upon what you showed us. So, considering that, I think I'll take a devil's advocate position and point out that myocardial swelling is a very difficult term to define with respect to intact heart. It is well known that no two mitochondria in a sample are alike, that is to say in structure; a fact that has been defined by a number of investigators is that they exist in at least two different conformations, orthodox and condensed. The whole situation is a paradox, in fact. We already know what these things mean; but to anyone, depending on where you sample, when you sample and the nutritional state of that particular tissue, the mitochondria can appear to be swollen and can appear to have no cristae, but in fact that is not true. They may just be changing shape in that localized area. I do not think at this stage of our experimentation that we can make any judgement that the section you are studying is indeed pathological, unless you actually see dissolved membranes.

Chairman: Would you be satisfied, Dr Schwartz, if there were a control series of six individuals, and we didn't know if they had alcohol or something else noxious?

Dr Schwartz: Only six? And it's a double blind experiment! You have many serial sections of the E.M. You give it to someone else working down the hallway and you have to divide it up. Yes, that would be a nice experiment.

DR BURCH: We gave some mice alcohol to drink; we gave beer to some, used $5 \%$ alcohol in water for others, and even 10 and $20 \%$ alcohol in aqueous solution for others. The mice drank the alcohol for 6 months before they were killed. Six months is a quarter of the life span of a mouse, equivalent to perhaps 20 years of a man's life; and the mice were drinking about one fourth of their body weight in alcoholic beverage, which is equivalent to a man drinking extremely heavily. Yet we noted very little change in the myocardium of the mouse. Thus, we cannot extrapolate from a mouse to man. Maybe mice can handle alcohol by some special metabolic mechanisms. We observed slight change in mitochondria. As $\mathrm{Dr}$ Schwartz has just said, the variations were great. But there was relatively little change in view of the tremendous amount of alcohol consumed by these animals. What this means for man, I do not know. The relationship of alcohol to malnutrition must be considered for alcoholic cirrhosis. 
How much of the damage is due to alcohol and how much is due to other factors remains unknown.

DR OLSEN: May I ask a question about your work on mice? They did show early changes of cristolysis. These were similar to the changes which were observed in the biopsies before and after alcohol. Accepting $\mathrm{Dr}$ Schwartz's comments on sampling, I think this is pretty convincing evidence that alcohol may well have a deleterious effect on the myocardium.

DR OAKLEY: I am easily persuaded that alcohol causes acute myocardial depression and that the physiological change is accompanied by morphological change which can be recognized. I have great difficulty in getting over the next jump, which is to link the acute myocardial depressant effect of alcohol with chronic heart failure and chronic congestive cardiomyopathy. There are many acute myocardial depressants. We have a right to assume the changes are transient, because otherwise those of us who do occasionally take alcohol would have a steadily accumulating deficit of myocardial function (and if I may revert to the previous paper of Dr Burch's, the same really goes for virus myocarditis). What is the evidence for any link between the acute effect and the chronic illness? Do either alcohol or viruses have any determinable association with the chronic disorder that we are discussing today?

Chairman: Well, perhaps we can return to this more general discussion later on, but now we have to go on to Dr Coltart's paper on the disposition of digoxin in the cardiomyopathic heart. 